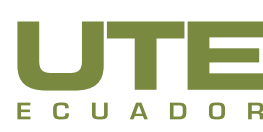

\title{
IMPACTO DEL PROCESO SENSORIAL EN EL APRENDIZAJE DE NIÑOS CON PARÁLISIS CEREBRAL ASOCIADO CON RETARDO MENTAL SEVERO
}

\author{
${ }^{1}$ Azucena Macías M, ${ }^{2}$ Sonia Arcos C.
}

${ }^{1}$ Fundación de Padres de Familia de Niños, Jóvenes y Adultos con discapacidades Psicomotrices Severas, FUDISE. Quito

- Ecuador, dra.azucena.macias@gmail.com

2Universidad Tecnológica Equinoccial, Quito - Ecuador, sac2192003@yahoo.com

Recepción / Received: 22, 08, 2014

Aceptación / Accepted: 10, 11, 2014

Publicado / Published: 12, 12, 2014

\section{Resumen:}

En este artículo se presenta el resultado de una investigación realizada en niños diagnosticados con Parálisis Cerebral (PC) asociado con Retardo Mental Severo (RMS). Para este propósito se asignó entrevistas, encuestas dirigidos a padres y personal de FUDISE (Fundación de Padres de Familia del Centro de Educación Especial) y un formato sensorial aplicado antes y después de un proceso multisensorial, que permite determinar la relación de este con el aprendizaje.

Mediante el trabajo realizado y considerando la puntuación alcanzada en las áreas sensoperceptivas, se evidenció que a pesar del daño motor y cognitivo de tipo severo que presentan los niños, existió un leve incremento del aprendizaje y se registró una mayor dificultad de abordaje de la propioceptiva, debido a que la severidad motora constituye un limitante de movimiento, postura y respuesta.

\section{Palabras clave:}

Aprendizaje, estimulación multisensorial, parálisis cerebral, procesos sensoriales, retardo mental severo.

\section{Abstract:}

This article presents the results of conducted research in children's diagnosed with Cerebral Palsy (CP) associated with Severe Mental Retardation (RMs) assigned for this purpose interviews, surveys aimed at parents and staff FUDISE (Parent Foundation of Special Education Center) and a sensory format applied before and after a multisensory process, which determines the relation of this to learning.

By considering the work done and the score achieved in the areas sensoperceptive is evidenced, despite the damage to motor and cognitive severe type presented by children's, there was a slight increase in learning more difficulty recording proprioceptive approach because that severity is a limiting motor movement, posture and response.

\section{Keywords:}

Learning, multisensory stimulation, cerebral palsy, sensory, severe mental retardation. 


\section{INTRODUCCIÓN}

Dentro de los trastornos neurológicos en pediátrica, la Parálisis Cerebral (PC) o disfunción motriz, constituye la discapacidad motora de la infancia con mayor porcentaje. La PC es una lesión que aparece en la primera infancia (desde la concepción hasta los 5 años). Según fuente bibliográfica dentro de la definición más aceptada propuesta por el comité de expertos en el 2005 acerca de lo que es la PC, se afirma: "...es un trastorno en el desarrollo del tono postural y del movimiento de carácter persistente, que condiciona una limitación en la actividad, secundario a una agresión no progresiva, a un cerebro inmaduro" (Lorente, 2007).

Al ser la PC estática y no progresiva, puede estar asociada con otras lesiones como déficit cognitivo en mayor o menor grado, trastornos sensoperceptivos, problemas auditivos visuales, lingüísticos, deglutivos, epilepsia entre otras.

La temática sobre los niños con discapacidades motoras e intelectuales y su atención en el Ecuador se ha ido modificando notablemente. Es así que de acuerdo con el Reglamento General de la Ley Reformatoria de Discapacidades (2003), en el art. 5, se ordena: "establecer un sistema educativo inclusivo para que los niños y jóvenes con discapacidad se integren a la educación general. En los casos en que no sea posible su integración, por su grado y tipo de discapacidad, recibirán la educación en instituciones especializadas, que cuenten con los recursos humanos, materiales y técnicos ajustados a sus necesidades para favorecer el máximo desarrollo posible" (Reglamento General a la Ley de Discapacidades, 2002).

Bajo la premisa de que la PC constituye un trastorno neurológico como secuela de lesiones del Sistema Nervioso Central (SNC); se establece que la población de estudio, presenta como diagnóstico médico una atrofia cortico, subcortical que da como resultado un tipo motor predominante como: diparesia espástica, cuadriparesia, hemiparesia e hipotonía, todas ellas asociadas con retardo mental severo.

Con este trabajo investigativo se pretende dar a conocer el impacto que tiene el aplicar los procesos multisensoriales como una estrategia terapéutica - educativa y la influencia que tienen en el aprendizaje de niños con diferentes condiciones motoras (PC), edades, géneros asociado con Retardo Mental Severo (RMS) en la Fundación de Padres del Centro de Educación Especial "FUDISE".

Para desarrollar este estudio se establecieron registros por medio de un test sensorial creado a través de varias investigaciones (pre y post) con ítems establecidos en las áreas visual, auditiva, táctil, olfativa, gustativa, propioceptivo y vestibular las cuales se basan en la obtención de valores que oscilan entre cero y cinco. Así pues, se puede sistematizar el grado de intensidad, el ritmo de aprendizaje y los cambios que se evidencien por leves que estos sean, luego de aplicar como metodología terapéutica - educativa a la estimulación multisensorial en todas las áreas anteriormente indicadas; ya que el aprendizaje se obtiene por una conexión mecánica entre estímulo - respuesta y la utilización de reforzadores, a través de rutinas; donde se busca que el sujeto con daño neurológico severo, utilice sus sentidos y la aptitud motora para establecer una relación con el medio que le rodea, para que sean lo más independientes posibles, tomando en consideración sus limitaciones tanto física como cognoscitivas.

Obtenidos los resultados de la investigación, se demuestra que a pesar de las deficiencias severas de estos pacientes, no constituyen un limitante para que ellos puedan aprender a través de programas multisensoriales en Sala Sensorial y Jaula de Rocher, con los cuales podrán satisfacer las necesidades prioritarias, al formar parte de un núcleo familiar y social.

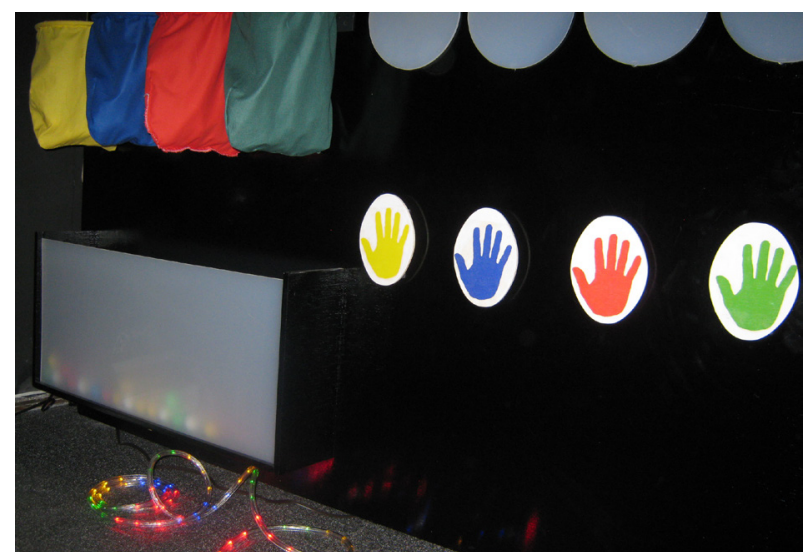

Figura $N^{\circ}$ 1. Sala sensorial.

Fuente: La autora

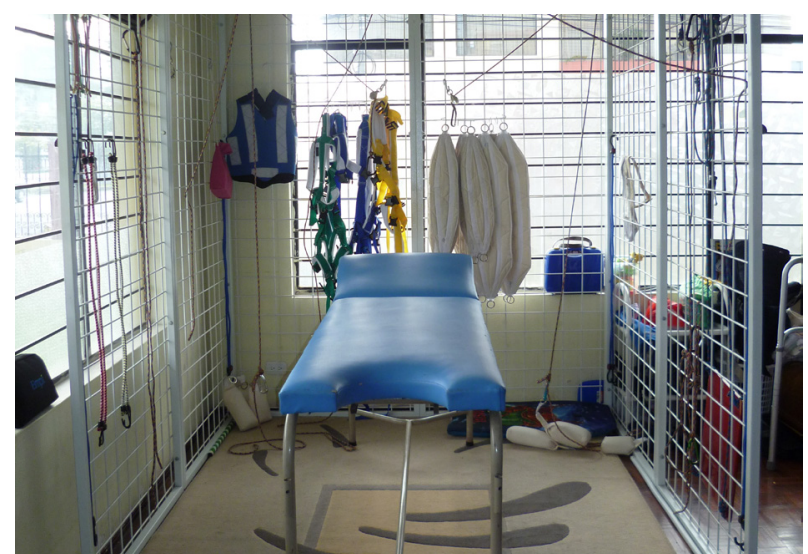

Figura N ${ }^{\circ}$ 2. Jaula de Rocher. Fuente: La autora 


\section{METODOLOGÍA}

Las personas que son diagnosticadas con PC asociado con RMS, presentan déficit sensorial, debido a que: "el cerebro no es capaz de integrar bien las sensaciones, y se ven afectadas muchas facetas de la vida cotidiana, por lo que el individuo debe realizar mayor esfuerzo en comparación con otros" (Ayres, 2008).

Por estarazón sehizo oportuno, establecercomo metodología terapéutico - educativa la estimulación multisensorial, que es una aliada por sus múltiples beneficios y por proporcionar, a través de los sentidos, un efecto positivo, puesto que activa las funciones cerebrales como la memoria a corto y largo plazo, la atención y la concentración, entre otras, al potenciar de esta manera el desarrollo cognitivo, mediante la aplicación de una buena y adecuada educación sensorial.

De acuerdo con lo expuesto anteriormente, el presente trabajo se fundamenta en una interrogante fundamental que consiste en conocer si los procesos multisensoriales influyen o no directamente en el aprendizaje de niños con PC asociados con RMS; si los canales sensoriales pueden ser abordados sin que las condiciones motoras sean un limitante, establecer cuales son las áreas sensoperceptivas más hábiles o las de mayor complejidad al ser abordadas, y cuál es el ritmo de aprendizaje de estos niños, de acuerdo con el daño motor que presentan.

La investigación se sustenta, desde el punto de vista filosófico y epistemológico, en un corte cuantitativo.

Para tener una visión más acertada sobre el proceso sensoperceptivo y la influencia que produce en el aprendizaje, se considera como alternativa fundamental la aplicación de la historia biopsicosocial que sirve para recoger la información sobre los datos acerca de los antecedentes personales como: proceso evolutivo (pre - peri y post natal), desempeño en actividades de la vida diaria (AVD) y socio familiares; la aplicación de pruebas psicométricas con las cuales proporcionen una edad de desarrollo aproximada además de llevar un monitoreo continuo y directo mediante un registro de las conductas más significativas: antes, durante y después del trabajo multisensorial (método de observación), a través del test sensorial (método de medición) con el cual se pudo comprobar el vinculo existente entre los procesos sensoriales y el aprendizaje de los niños diagnosticados con PC asociado con RMS (método experimental).

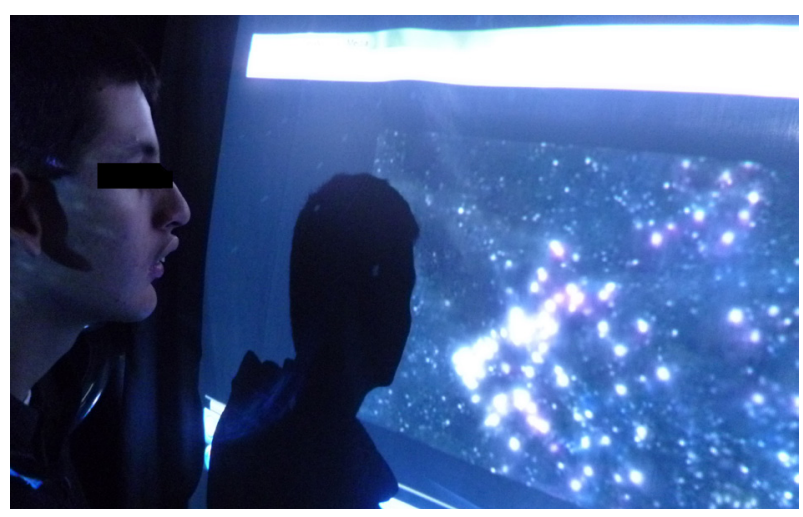

Figura $N^{\circ} 3 a$. Estimulación multisensorial, visual. Fuente: La autora

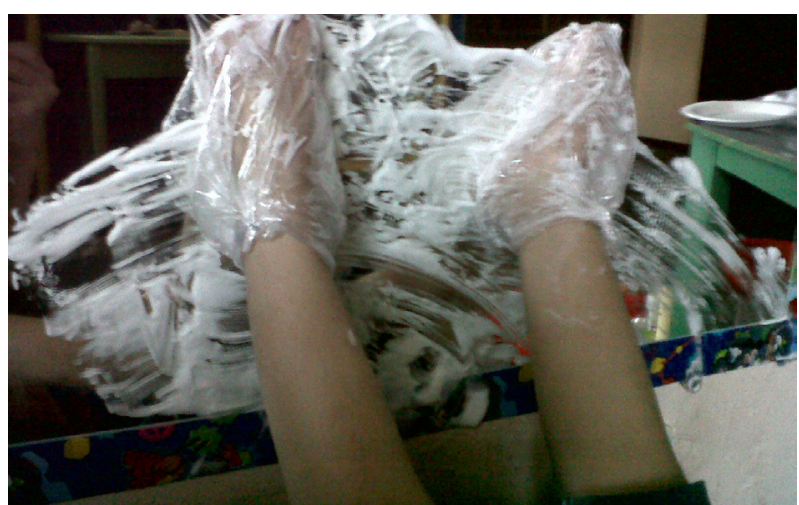

Figura №3b. Estimulación multisensorial, táctil. Fuente: La autora

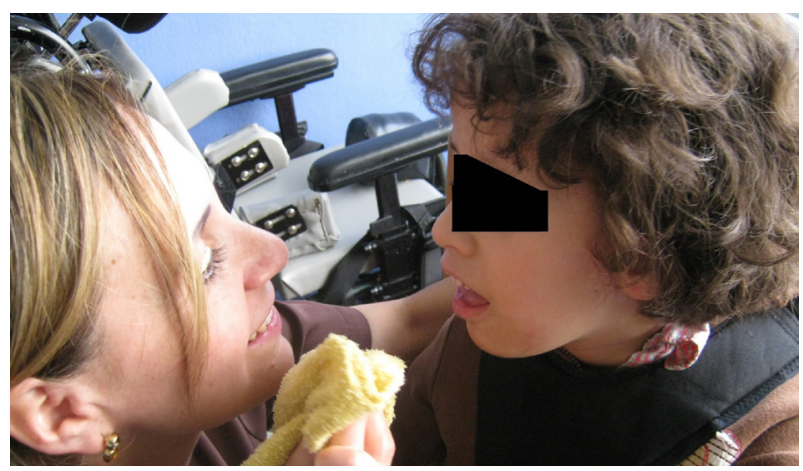

Figura Nº 3c. Estimulación multisensorial, olfativa. Fuente: La autora

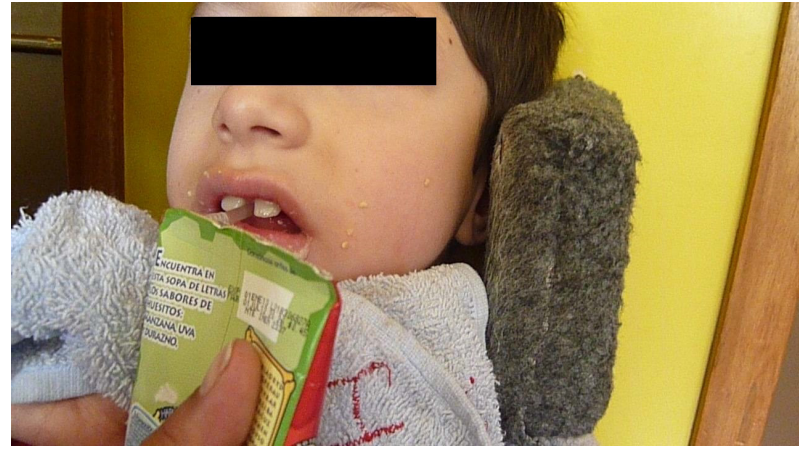

Figura $N^{\circ} 3 d$. Estimulación multisensorial, gustativa. Fuente: La autora 


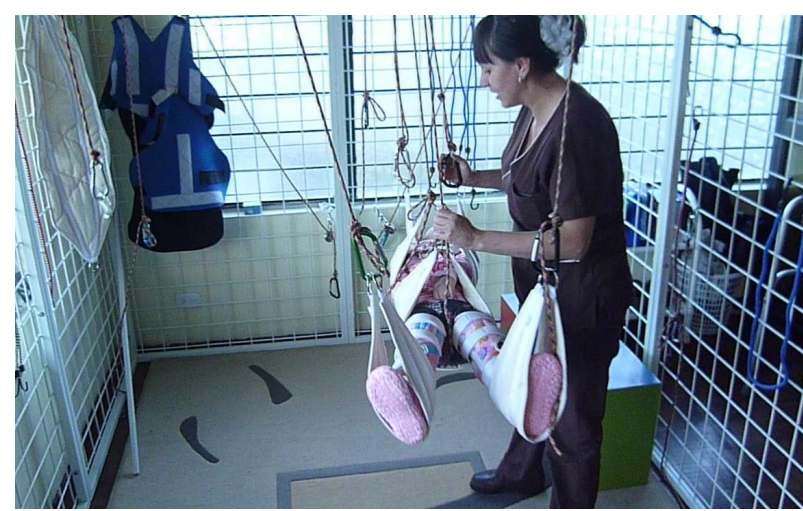

Figura №3e. Estimulación multisensorial, vestibular. Fuente: La autora

\section{ANÁLISIS E INTERPRETACIÓN DE DATOS}

La población de estudio estuvo conformada por 14 sujetos con daño neurológico (PC asociado con RMS) de diferentes géneros y edades (Fig. 4), a los que se les aplicó una metodología terapéutica - educativa basada en la estimulación multisensorial, con la finalidad de establecer qué impacto tienen estos procesos en el aprendizaje, hipótesis planteada al inicio de la investigación. Para su comprobación se utilizó la prueba de chi cuadrado, con un nivel de significación de $\mathrm{a}=0.05$, y para determinar la independencia entre las variables se consideró el valor de $\mathrm{P} \geq \mathrm{a}$.

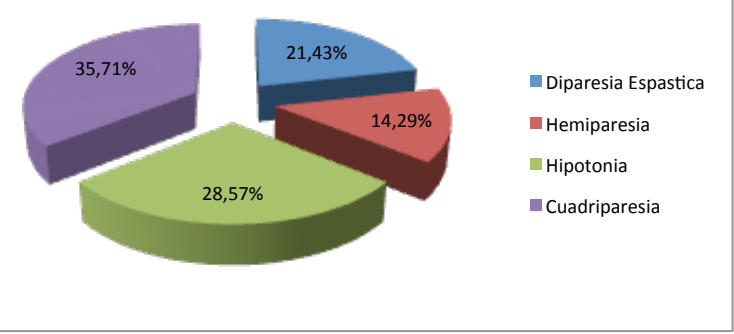

Figura № 4 . Tipificación de la población de estudio. Fuente: La autora

El tipo de PC que presenta un paciente depende de la zona de cerebro afectada (lesión que ocasiona un desequilibrio funcional dando como resultante un tipo de trastorno motor. En todos los casos, se registró un daño mental severo.

\section{Resultados de aprendizajes:}

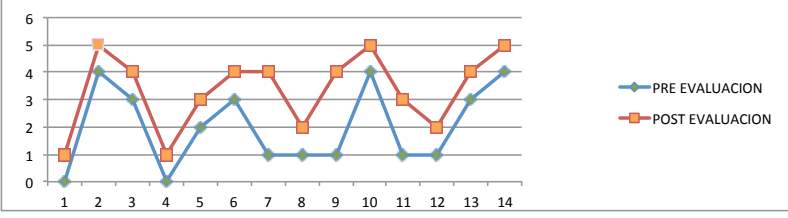

Figura $N^{\circ} 5$. Pre y post evaluación con respecto a la percepción visual. Fuente: La autora

El propósito de la percepción visual es proveer al niño/a de la información necesaria utilizando una gama de luces, colores, formas, etc., estimulando de esta manera su mecánica funcional e integrando gradualmente la información visual con la finalidad de interactuar con el entorno físico. Obtenidos los resultados (Fig. 5), se puede apreciar que al existir un daño motor e intelectual de cada uno de los pacientes, hay un incremento en la parte perceptiva visual, y son los diparéticos y los hipotónicos los que alcanzan un mejor desempeño.

El enfoque del proceso de sensopercepción auditiva, trabaja la parte de atención, organización, discriminación y ubicación de fuente sonora, las que ayudan almacenar la información en la memoria a través de las experiencias vividas. Favorecen la adquisición de la función de alerta y la social. Al establecer un análisis (Fig. 6) luego de aplicada la estimulación auditiva, se verifica que cinco de los casos se mantienen dentro del mismo parámetro, mientras que encontramos un pequeño incremento en los cuadriparéticos, seguidos por hipotónicos, diparéticos y hemiparéticos.

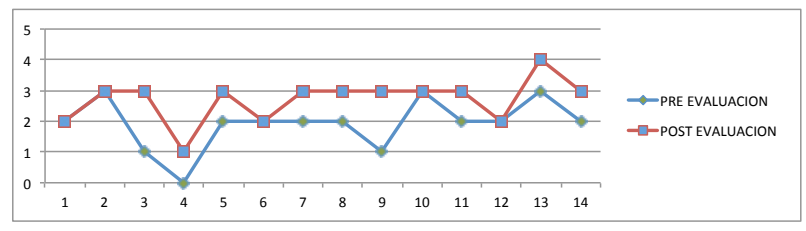

Figura $N^{\circ} 6$. Pre y post evaluación con respecto a la percepción auditiva. Fuente: La autora

El córtex parietal posterior desempeña un papel importante en la integración de las sensaciones táctiles y considerando que mientras cuanto antes se empiece a estimular y a potenciar al máximo el proceso perceptivo al niño/a, utilizando un material que disponga de características táctiles suficientemente contrastadas y diferenciadas, estas marcarán su vida. Con respecto a la percepción táctil (Fig. 7), se estima que el incremento alcanzado se consigue de manera leve en aquellos pacientes diagnosticados con hipotonía, cuadriparesia, diparesia y hemiparesia; se mantienen ocho de los casos con un ligero y mediano aprendizaje.

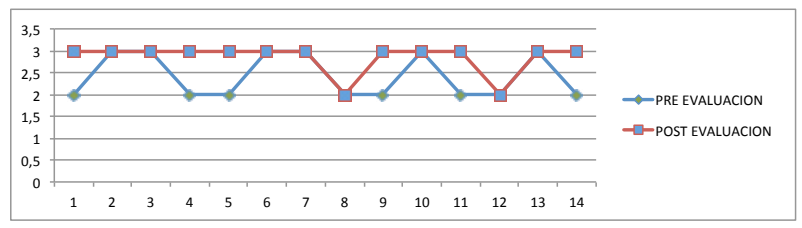

Figura $N^{\circ}$ 7. Pre y post evaluación con respecto a la percepción táctil. Fuente: La autora

La función del olfato es discriminar diferentes olores (en contraste), sean estos agradables - desagradables, suaves - fuertes, conocidos - desconocidos; siendo capaz de acuerdo a su lesión motora será capaz de rechazar, apartarse o fruncir el ceño si un olor no es de su agrado; y logrará que posteriormente lo relacionen con actividades de la vida diaria. Determinada la tabla estadística sobre la percepción olfativa (Fig. 8), se observa que gran parte de 
los sujetos de estudio se mantienen, considerando que poseen un nivel que oscila entre un aprendizaje medio y alto, seguido por un avance mínimo en el caso de diparesia, cuadriparesia, hemiparesia e hipotonía.

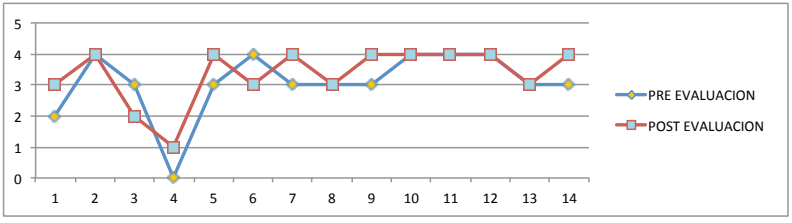

Figura $N^{\circ} 8$. Pre y post evaluación con respecto a la percepción olfativa. Fuente: La autora

El sentido del gusto depende de la estimulación de las papilas gustativas, situadas en la lengua y en el paladar, a través de los alimentos (dulces, salados, ácidos, umami, etc.), transmitiendo la información hasta el lóbulo temporo - occipital del cerebro. Cabe mencionar que, a más de discriminar sabores y texturas, se estimula esta área para desarrollar los movimientos oro - faciales (chupar, masticar, deglutir). En base al análisis (Fig. 9) se especifica que seis casos se mantienen en un buen índice y ocho incrementan su nivel de aprendizaje (cuadriparesia (3), hemiparesia (2), hipotonía (2) y diparesia (1).

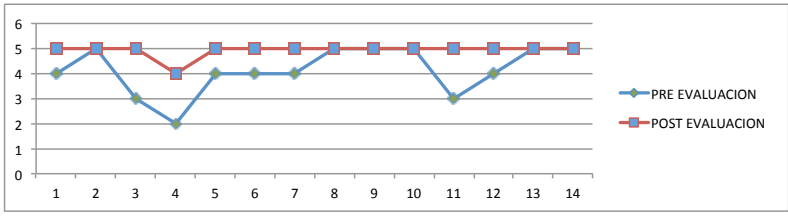

Figura № 9. Pre y post evaluación con respecto a la percepción gustativa Fuente: La autora

Para realizar un movimiento se requiere de la correcta integración entre la información sensitiva de los músculos, tendones y ligamentos, así como del SNC como órgano efector de la respuesta motora. Este proceso no se ejecuta de manera apropiado en las personas diagnosticadas con daño neurológico (PC), donde presentan un funcionamiento anormal en alguno de estos componentes, lo que da como resultado un movimiento desorganizado: es por esta razón de que solo cuatro pacientes alcancen un leve incremento; mientras que el resto permanece en ausencia total (Fig.10).

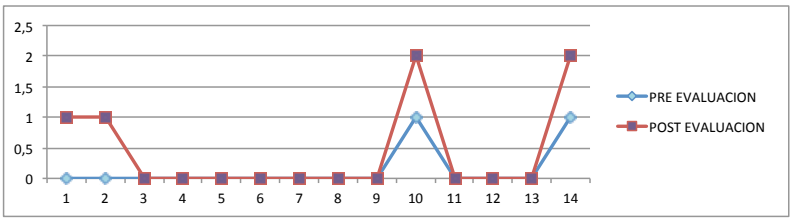

Figura № 10. Pre y post evaluación con respecto a la percepción propioceptivo.

Fuente: La autora

El propósito de la estimulación vestibular es ayudar al paciente a elaborar las respuestas adecuadas ante un estimulo organizado y sistemático del movimiento en cadena que estimule el cerebro. De acuerdo con los resultados (Fig.
11), se puede apreciar que dentro de esta área se muestra un aprendizaje leve, si se considera que la población de estudio presenta dificultades para mantener la cabeza erguida, sentarse sin apoyo, problemas en la marcha independiente, deficiencia de equilibrio y coordinación, etc.

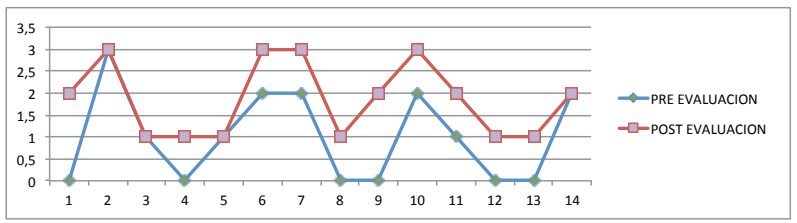

Figura $N^{\circ} 11$. Pre y post evaluación con respecto a la percepción vestibular. Fuente: La autora

El fomentar la estimulación multisensorial en este tipo de pacientes hace que mejoren su calidad de vida, ya que es el canal para que se puedan conectar consigo mismo y con el entorno. Establecida la estadística en donde se puede comparar los resultados alcanzados de manera global en cada uno de los sujetos (Fig. 12), podemos concluir que luego de aplicar una estimulación sensorial como estrategia metodológica nueve (9) de los pacientes muestran un aprendizaje leve y cinco (5) de ellos se mantienen.

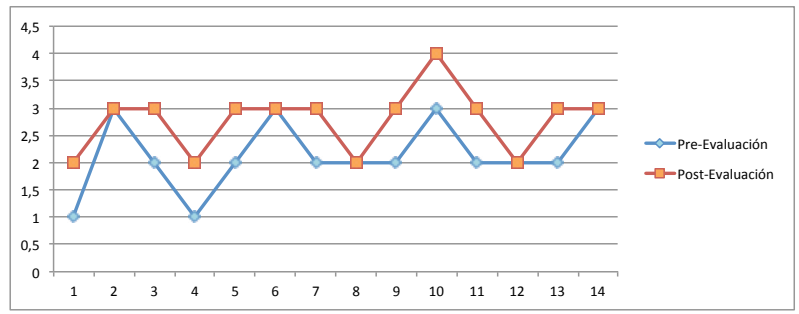

Figura Nº 12. Evaluación multisensorial (PRE - POST). Fuente: La autora

\section{CONCLUSIONES}

La sala multisensorial es un espacio que se adapta a las necesidades, intereses y posibilidades de cada niño, cuyo objetivo según Gómez (2009) es "la integración de los sentidos y la mejora de la calidad de vida en todas aquellas personas con déficit físico o psíquico especifico".

La utilización de programas multisensoriales destinadas al tratamiento de niños con lesiones cerebrales, que se han realizado en otros países, como es el caso del estudio publicado por la revista Medical Journal de la Universidad de Miami, en el centro de rehabilitación Jackson Memorial, demuestran que con la creación de un programa multisensorial los resultados son muy alentadores, tanto en la educación como en enfoques terapéuticos. Informes documentados muestran casos de niños con discapacidad que han mejorado su calidad de vida por el uso de una estimulación dosificada o controlada que le permite percibir sensaciones varias que ayudan a adquirir el aprendizaje por 
medio del descubrimiento.

El interés del estudio investigativo fue conocer el impacto que existía en el aprendizaje al aplicar una metodología terapéutica - educativa basada en la estimulación multisensorial de una población conformada por 14 niños con daño neurológico severo (PC asociado con RM) de diferentes géneros y edades.

Luego del proceso se puede determinar que entre las evaluaciones del pre y post test realizadas, sobre las sensopercepciones y su impacto en el aprendizaje, se obtiene como valor $\mathrm{P}=0.068$ y este valor es mayor que a (nivel de confianza 0.05); por lo tanto queda comprobado la hipótesis de que los procesos multisensoriales aplicados como metodología terapéutica - educativa, influyen directamente en el aprendizaje de niños/as con PC asociados con RMS, de "FUDISE".

De acuerdo a los resultados de aprendizaje obtenidos en las áreas sensoperceptivas encontramos en lo referente al número de niños: visual (14 casos), vestibular (10 casos), auditiva (9 casos), gustativa (8 casos), olfativa (7 casos), táctil (6 casos) y propioceptivo (4 casos), que dependiendo de la modalidad de intervención multisensorial utilizada, nueve de ellos adquieren un aprendizaje que oscila entre ligero (2 casos) - medio (6 casos) - alto (1 caso), mientras el resto se mantiene.

Se pudo determinar que dentro de todas las áreas sensoriales que se abordaron, la propioceptiva tuvo la menor puntuación, debido a que la severidad motora es un limitante significativo para el movimiento y la postura (ubicación corporal en el espacio), así como también, el déficit cognitivo (reconocimiento corporal). Todas ellas fueron las causantes de un impacto negativo directo en el aprendizaje. Es importante recalcar que el manejo del niño con un compromiso no solo motor; sino también con déficit cognitivo y alteraciones sensoriales, depende de un tratamiento interdisciplinario, en el que se logre cubrir, a través de la aplicación sensorial, las diferentes áreas de necesidad del sujeto. Finalmente, puedo aportar desde mi experiencia, que esto no es un limitante para que aprendan los niños con PC asociados con RMS.

\section{RECOMENDACIONES}

1.- Establecer un programa sistematizado de abordaje multisensorial a desarrollarse no solo en FUDISE, sino también en otras instituciones en donde se trabaje con este tipo de daño severo, pues los resultados obtenidos nos indican que estos impactan directamente en el aprendizaje de niños/as con PC asociado con RMS.

2.- Utilizar como canales de acceso multisensorial prioritario las vías que obtuvieron mejor resultado con impacto en el aprendizaje (visual, vestibular, auditiva, gustativa, olfativa, táctil).

3.- Potenciar el abordaje del área sensorial deficiente o que presentó menor porcentaje de resultado, en referencia al aprendizaje propioceptivo, con la finalidad de alcanzar en lo posible un óptimo funcionamiento.

4.- Reforzar el manejo de cuidado postural y alineación biomecánica al momento de iniciar un proceso de estimulación multisensorial en la vía propioceptiva, pues esta obtuvo puntuaciones casi nulas, debido a la complicación de movimiento, postura y respuesta.

5.- Crear vías de comunicación e información a los padres, concienciando que los niños/as con PC asociado con RMS, tienen potencial de aprendizaje y deben ser incluidos en un programa con base en estimulación sensorial.

6.- Implementar un sistema organizado de estimulación multisensorial, en el cual se realicen evaluaciones y reevaluaciones periódicas, cada seis meses, y llevar durante todo este proceso, registros audio - visual y cuali cuantitativos del progreso.

Estoy convencida de que la atención multisensorial en pacientes con problemas motores asociados con un déficit cognitivo incrementan su desarrollo. Por lo tanto, dejo una apertura para que se siga investigando sobre esta temática cuyos aportes serán de gran utilidad para todas aquellas personas con daño neurológico severo, puesto que ellos tienen los mismos derechos que cualquier otro individuo de adquirir un aprendizaje a través de una educación especializada que mejore significativamente su calidad de vida.

\section{REFERENCIAS BIBLIOGRÁFICAS}

Ayres, J. A. (2008). La Integración Sensorial en los Niños. Madrid: Editorial TEA.

Gómez Gómez, María del Carmen (2009). Aulas Multisensoriales en Educación Especial Estimulación e Integración Sensorial en los Espacios Snoezelen (1. edición) Madrid - España. Editorial Ideas propias S.L., Vigo, pàginas 7-9.

Lorente Hurtado, I. (2007). La parálisis cerebral. Actualización del concepto, diagnóstico y tratamiento. Barcelona: Unidad de Neuropediatría. Servicio de Pediatría. Hospital Sabadell.

Reglamento General a la Ley de Discapacidades (2002). Recuperado de http://dredf.org/legal-advocacy/ international-disability-rights/international-laws/ ecuador-reglamento-general-a-la-ley-dediscapacidades/ 